\title{
Diagnostic Approaches for Invasive Aspergillosis-Specific Considerations in the Pediatric Population
}

\author{
Thomas Lehrnbecher $^{1 *}$, Angela Hassler ${ }^{1}$, Andreas H. Groll ${ }^{2}$ and Konrad Bochennek ${ }^{1}$ \\ ${ }^{1}$ Division of Pediatric Hematology and Oncology, Hospital for Children and Adolescents, Johann Wolfgang Goethe-University, \\ Frankfurt, Germany, ${ }^{2}$ Infectious Disease Research Program, Center for Bone Marrow Transplantation, Department of \\ Pediatric Hematology/Oncology, University Children's Hospital, Münster, Germany
}

OPEN ACCESS

Edited by:

Juergen Prattes,

Medizinische Universität Graz, Austria

Reviewed by:

Volker Strenger,

Medizinische Universität Graz, Austria

Murat Akova,

Hacettepe University School of

Medicine, Turkey

*Correspondence:

Thomas Lehrnbecher

thomas.lehrnbecher@kgu.de

Specialty section:

This article was submitted to Fungi and Their Interactions,

a section of the journal

Frontiers in Microbiology

Received: 12 January 2018 Accepted: 07 March 2018 Published: 26 March 2018

Citation:

Lehrnbecher T, Hassler A, Groll AH and Bochennek K (2018) Diagnostic Approaches for Invasive Aspergillosis-Specific Considerations in the Pediatric Population.

Front. Microbiol. 9:518.

doi: 10.3389/fmicb.2018.00518
Invasive aspergillosis (IA) is a major cause of morbidity and mortality in children with hematological malignancies and those undergoing hematopoietic stem cell transplantation. Similar to immunocompromised adults, clinical signs, and symptoms of IA are unspecific in the pediatric patient population. As early diagnosis and prompt treatment of IA is associated with better outcome, imaging and non-invasive antigen-based such as galactomannan or B-D-glucan and molecular biomarkers in peripheral blood may facilitate institution and choice of antifungal compounds and guide duration of therapy. In patients in whom imaging studies suggest IA or another mold infection, invasive diagnostics such as bronchoalveolar lavage and/or bioptic procedures should be considered. Here we review the current data of diagnostic approaches for IA in the pediatric setting and highlight the major differences of performance and clinical utility of the tests between children and adults.

Keywords: Aspergillus, child, cancer, diagnostics, galactomannan, B-D-glucan, polymerase chain reaction, imaging

\section{INTRODUCTION}

Invasive fungal disease (IFD), in particular invasive aspergillosis (IA), is a major cause of morbidity and mortality in immunocompromised children and adults (Pagano et al., 2007; Sung et al., 2007; Upton et al., 2007). The definite diagnosis of IA ("proven aspergillosis") is extremely difficult and requires the visualization of the organism in tissue or its microbiological detection in an otherwise sterile compartment. In daily clinical practice, the diagnosis of IA is mostly made by appropriate imaging studies and diagnostic biomarkers in blood and other readily available specimens ("probable or possible aspergillosis"), and these methods are important guiding decisions about the institution and the choice of antifungal agents as well as regarding the duration of therapy. The rationale for this clinical practice is the fact that early institution of appropriate antifungal agents is the factor that is most strongly associated with favorable outcome (Caillot et al., 1997).

Different strategies have been developed in order to decrease the incidence of IA and to improve outcome. Many experts in the field administer prophylactic mold-active antifungals to patients at high-risk for IA. In the pediatric setting, this population includes patients with acute myeloid leukemia (AML), relapsed acute leukemia [both AML and acute lymphoblastic leukemia (ALL)] 
as well as children undergoing allogeneic hematopoietic stem cell transplantation (HSCT), all of which have a risk $>10 \%$ for IA (Sung et al., 2007; Hol et al., 2014; Fisher et al., 2017). The situation is more complex in children undergoing therapy for ALL, the largest patient population in pediatric hematology. Depending on the protocol used and some additional factors such as age, the presence of prolonged bone marrow failure, and concomitant use of corticosteroids, some of these patients are at high risk for IA, whereas others are at low risk; unfortunately, however, these subgroups are poorly defined to date (Groll et al., 2014). In contrast, children undergoing autologous HSCT and children diagnosed with non-Hodgkin and Hodgkin lymphoma as well as children with solid tumor have a low risk for IA, although IA is sometimes seen in these patients (Groll et al., 2014). Some institutions may not use antifungal prophylaxis but screen afebrile and asymptomatic neutropenic patients at high-risk for IA using non-culture based methods, and institute mold-active compounds only if the patient develops fever and screening tests turn positive ("pre-emptive therapy"). However, pediatric data of pre-emptive therapy are scarce, and therefore, in children, the approach to use empirical antifungal therapy is more common. With this concept, antifungals are started in all high-risk neutropenic patients who develop fever which does not respond to broad spectrum antibiotics.

The result of a diagnostic tool is not only important in the decision to start antifungal therapy, but may also help to modify and guide the use of antifungals. Although, as compared to studies with adult patients, considerably less data are available in the pediatric population, it has been recognized that the performance and the usefulness of diagnostic tools to detect IA may differ between children and adults. This has led to several pediatric specific guidelines for the diagnosis and treatment of IFD (Groll et al., 2014; Science et al., 2014; Lehrnbecher et al., 2017b), and at present, the European Organisation for Research and Treatment of Cancer (EORTC) and the Mycosis Study Group (/MSG) (EORTC/MSG) consensus definitions for the diagnosis of IFD undergo a second revision in which pediatric specific diagnostic considerations will be included. The present review will focus on the value of diagnostic tests to detect IA in the pediatric setting and highlight major differences between children and adults.

\section{CLINICAL SIGNS AND SYMPTOMS OF INVASIVE ASPERGILLOSIS}

Similar to adults, clinical signs, and symptoms of IA in children are often non-specific and cannot be readily distinguished from other infections caused by bacteria, viruses, and other fungi or from certain non-infectious complications caused by the underlying disease or sequelae of its treatment. Importantly, fever during neutropenia which is unresponsive to broad-spectrum antibiotics may be the only sign of IA. Other clinical symptoms depend of the site of infection: as IA most often involves the lung, patients may complain about pleural or back pain or dyspnea, whereas headache, altered mental state, seizure, or focal neurologic signs may indicate central nervous system (CNS) involvement (Hassler et al., 2015; Lehrnbecher et al., 2017a).

\section{MICROSCOPY AND FUNGAL CULTURE}

Overall, standard diagnostic techniques such as microscopy and culture to detect fungal organisms do not differ between children and adults. A microbiologist experienced in the diagnosis of fungal infections should microscopically examine all samples from invasive diagnostic procedures such as bronchoalveolar lavage (BAL) or biopsies (Ruhnke et al., 2012). Special "fungal stains" such as periodic acid-Schiff, Grocott's methenamine silver, or optical brighteners (e.g., calcofluor white) are recommended to visualize hyphae. Despite the fact that microscopy can already provide important information (e.g., presence of septa, hyphal diameter, ramification pattern), a reliable differentiation between Aspergillus spp. and other filamentous fungi is impossible; in individual situations, although not standardized, and validated, immunohistochemical examination may be helpful (Hayden et al., 2003).

Although Aspergillus species are rarely recovered from the blood, all clinical samples from children who are at high risk for invasive fungal infections have to be cultured for fungi (Kontoyiannis et al., 2000). Blood samples and cerebrospinal fluid (CSF) should be processed immediately, and issue specimens have to be kept moist and must not be placed in fixatives. Importantly, negative culture results do not reliably exclude IA. If Aspergillus can be cultured, the isolate should be identified at the species level and antifungal susceptibility testing should be performed as this might impact therapy (Ruhnke et al., 2012).

\section{FUNGAL BIOMARKERS}

Due to the difficulties in isolating and culturing Aspergillus, there has been much effort to develop and evaluate various non-culture based assays. These assays include the serological detection of fungal antigens to support the diagnosis of IA in immunocompromised patients.

\section{GALACTOMANNAN}

Galactomannan (GM) is a carbohydrate constituent of the cellwall of Aspergillus spp. and is released by the fungus during cell growth. An FDA-approved enzyme immunoassay which employs the $3-1-5$ galactofuranosyl specific EB-A2 rat monoclonal antibody has been evaluated in the clinical setting to detect the molecule (Platelia ${ }^{\mathrm{TM}}$ Aspergillus Enzyme Immunoassay, BioRad). A number of studies evaluated the usefulness of GM as screening test in asymptomatic neutropenic adults (Maertens et al., 1999, 2001, 2004, 2005; Sulahian et al., 2001). Specificity and sensitivity of a positive test result were between 90 and 100\% and 80 and $100 \%$, respectively, and the negative predictive value (NPV) was greater than $90 \%$. More importantly, positive test results were seen before patients developed clinical symptoms 
of IA or before abnormalities could be detected in a chest CT scan (Hayden et al., 2003). The EORTC/MSG consensus group included positivity of GM assessed in serum, BAL fluid and CSF in their revised definitions of IFD (De Pauw et al., 2008). In serum, a cut-off of 0.5 is recommended by the manufacturer, whereas a cut-off of $\geq 1.0$ might be more appropriate for BAL fluid (Maschmeyer et al., 2015). In the adult setting, a number of guidelines recommend the use of GM for the diagnosis and management of IA (Maertens et al., 2010; Ruhnke et al., 2012; Maschmeyer et al., 2015).

A recent systematic review has scrutinized the available data of serum GM in pediatric cancer patients and children undergoing HSCT (Lehrnbecher et al., 2016). Eligible for the analysis were studies which included only patients up to 25 years of age, included at least $50 \%$ of patients suffering from cancer or undergoing HSCT, used EORTC/MSG criteria to define IA, and included patients without IA as a control group. The authors analyzed the test characteristics of 10 studies in which GM was used for screening and of eight studies which evaluated GM as a diagnostic tool, e.g., in patients with prolonged febrile neutropenia or in patients with pulmonary signs and symptoms. In the screening setting, a total of pediatric 688 patients were included, with the number of patients ranging between 17 and 198 per study; and in the diagnostic setting, 733 pediatric patients were included with a range of 38-145 patients per study. The prevalence of proven and probable IA ranged from 0.5 to 30.4 in the screening setting and from 0.0 to 30.8 in the diagnostic setting, respectively. Specificities and sensitivities ranged from 50 to $100 \%$ and from 0 to $100 \%$ in the screening setting and from 35 to $100 \%$ and from $14 \%$ to $100 \%$ in the diagnostic setting, respectively. Pooled specificity and sensitivity in the screening setting were $91 \%$ [95\% confidence interval (CI), 86-94\%] and $68 \%$ (95\% CI, $51-81 \%$ ), and $85 \%$ (95\% CI, 51-97\%), and $89 \%$ (95\% CI, 79-95\%) in the diagnostic setting, respectively. In adults, a meta-analysis has reported comparable data with a specificity of $81 \%(95 \%$ CI $72-90 \%)$ and a sensitivity of $82 \%$ (95\% CI 73-90\%) (Maertens et al., 2005). Positive predictive values (PPVs) in children were rather low and ranged between 0 and $100 \%$ in each setting, while the negative predictive values (NPVs) were considerably higher and ranging from 85 to $100 \%$ and from 70 to $100 \%$ in the screening and diagnostic setting, respectively. It is well known that there are a number of reasons for false-positive results, such as the concomitant use of some batches of beta-lactam antibiotics or the cross-reactivity of the assay with other fungi (e.g., Penicillium spp., Fusarium spp., Histoplasma capsulatum, Cryptococcus neoformans) (Viscoli et al., 2004). On the other hand, the use of mold-active prophylaxis may result in a high rate of false-negative results (Lass-Florl, 2017), although this could not be proven in the meta-analysis of pediatric studies as the antifungal prophylaxis was not standardized in most of the included studies. It is important to note that predictive values were similar when pediatric patients with possible IFD were classified as not having IFD or when patients with possible IFD were excluded from analysis. Similarly, predictive values were comparable in both screening and diagnostic testing setting, and test characteristics did not systematically improve as disease prevalence increased. Based on the data of this meta-analysis, recent recommendations of an international expert panel recommended to consider not using GM testing in children with prolonged febrile neutropenia (weak recommendation, moderate quality of evidence), because (1) due to the poor PPV, the clinical consequence based on test results is often incorrect (e.g., to institute mold-active antifungals) and (2) despite the high NPV, a negative GM test does not rule out non-Aspergillus molds, which clearly limits the value of the assay. However, as the diagnosis of IA is extremely difficult and does not depend on a single parameter alone, and as the local epidemiology plays an important role, GM testing may have an impact in daily clinical decision making with consideration of its limitations.

It is important to mention that the design of the available studies is in part highly problematic, as many used GM as the reference parameter to define IA. Similarly, in studies which required two positive samples to define a positive test result of GM, it often remains unclear during which time period the two samples had to be drawn. Another problem causing heterogeneity of the results of different studies is the variable aggressiveness to use $\mathrm{CT}$ scans or the invasive diagnostic procedures to prove or to exclude IA, all of which may bias the results.

Limited pediatric data are available on the assessment of GM in BAL. One study retrospectively analyzed the GM results in BAL fluid, which was obtained in 85 children (59 among them were immunocompromised) (Desai et al., 2009). Proven IA was diagnosed in three patients, probable IA in 6, whereas 39 children had possible IA and 39 no evidence of fungal disease. Receiver-operating characteristics demonstrated that in immunocompromised children, a BAL GM cut-off value of 0.87 resulted in a sensitivity, specificity, PPV and NPV for probable/proven IA of 78, 100, 58, and $96 \%$, respectively. Although similar results were reported in another retrospective analysis of 72 bronchoscopies performed in immunocompromised children, the study demonstrated that the result of the GM assay in BAL had only little impact on the clinical decision, as in a considerable number of patients antifungal agents were continued or even started despite a negative GM result (de Mol et al., 2013); however, the duration of antifungal therapy was not considered in this analysis. Although colonization might be a bias for positive results of GM in BAL fluid, the promising results in children are supported by adult data on the use of GM testing in BAL fluid in patients in whom pulmonary aspergillosis was suspected (Maertens et al., 2009).

To date, relatively little data are published on the utility of GM testing in the CSF in order to diagnose CNS aspergillosis. A 18-months-old boy was reported with pulmonary and cerebral aspergillosis, in whom GM in the CNS was elevated (Roilides et al., 2003). In addition, one small case series reported on five patients with probable CNS aspergillosis (Viscoli et al., 2002). In these patients, GM levels in the CNS were significantly higher than GM levels of 16 control patients, suggesting the potential value of GM in CSF. Similarly, the testing of GM in urine specimens allows an easy and non-invasive sample collection, which would be interesting in particular in neonates and young children. In this respect, a recent study reported on promising 
results of measuring the urine $\mathrm{GM} /$ creatinine ratio in 71 adult patients with underlying hematological malignancies, but these results have to be validated in a pediatric patient population (Reischies et al., 2016).

Finally, there is growing interest in using serum GM as a surrogate marker for the effectiveness of antifungal therapy (Nouér et al., 2011; Chai et al., 2012; Kovanda et al., 2017). For example, one clinical trial in 158 adult patients demonstrated that an increase of the GM index of more than 0.25 from baseline by day 7 was associated with a significant increase of death compared to patients in whom the GM index increased less than 0.25 (Kovanda et al., 2017). Although not shown in the pediatric population, GM could individualize antifungal therapy as increases by day 7 could trigger treatment changes.

\section{1-3- $\beta$-D-GLUCAN}

As compared to GM, the use of 1-3-B-D-glucan (BG) as a biomarker allows the detection of a wider array of pathogenic fungi, including Aspergillus spp, Candida spp, Fusarium spp, Trichosporon, or Pneumocystis jirovecii since BG is present in the cell wall of many fungi. However, BG remains low or even negative in patients with cryptococcosis or mucormycosis. Positive values of $\mathrm{BG}$ can also be assessed during various bacterial infections, for example in infections due to Streptococcus pneumoniae or Pseudomonas aeruginosa, and additionally, BG may be detected in healthy individuals (Oz and Kiraz, 2011). Similar to GM, the EORTC/MSG consensus group included BG as mycological criterion in the revised definitions of IFD (De Pauw et al., 2008). A meta-analysis in adult patients demonstrated that different BG test assays have a similar performance [e.g., Fungitell ${ }^{\mathrm{TM}}$ (Associates of Cap Cod, Inc. Falmouth, MA), Fungitec-G (Seikagaku)], and that BG might be a useful tool to exclude IFD in the clinical setting, as for two consecutive tests, sensitivity and specificity were 50 and 99\%, respectively, and estimated PPV and NPV for an IFD prevalence of $10 \%$ were $84 \%$ and 95\%, respectively (Lamoth et al., 2012). However, it has to be noted that the assay has major limitations due to multiple factors which may cause false-positivity. For example, in addition to contamination, bacteremia, severe mucositis, the infusion of platelets with leukocyte-removing filters, the administration of albumin and immunoglobulins, or the administration of antibiotics such as amoxicillin-clavulanate or piperacillin-tazobactam have all been described as potential cause for false-positive results (Koltze et al., 2015). In addition, positive BG levels have to be interpreted with caution in patients who have had prior transient candidemia or have mucosal colonization with Candida species (Naselli et al., 2015).

There are limited data on the use of BG in children. It has been shown that mean values of BG are higher in normal healthy children than in healthy adults (Smith et al., 2007). It was suggested that the optimal cut-off level to identify neonates with invasive candidiasis was $125 \mathrm{pg} / \mathrm{ml}$ as compared to the $80 \mathrm{pg} / \mathrm{ml}$ recommended for adults, which resulted in a sensitivity and specificity of 84 and 75\%, respectively (Goudjil et al., 2013). A total of three studies evaluated BG in children receiving therapy for a malignancy or undergoing HSCT, among them 38 patients with proven/probable IFD (Zhao et al., 2009; Badiee et al., 2012; Koltze et al., 2015). Overall, wide ranges were found for specificity (29-82\%), sensitivity (50$83 \%)$, PPV (17-49\%), and NPV (84-96\%), respectively. Due to the limited pediatric data and the poor performance of the test in this patient population, BG testing is currently not recommended in the pediatric population as a routine diagnostic test, although it may be helpful under certain circumstances under awareness of its limitations (Groll et al., 2014; Lehrnbecher et al., 2017b).

\section{MOLECULAR DIAGNOSTIC TOOLS}

Polymerase chain reaction (PCR) has become a promising diagnostic tool for the early detection of IA. However, the method is not included to date as mycological criterion in the current EORTC/MSG definitions for IFD. This is due to a number of methodological issues, such as the optimal clinical sampling (e.g., whole blood, serum, plasma), the best DNA extraction method, and the optimal primer design (De Pauw et al., 2008; White et al., 2010, 2015b). In addition, multiple PCR approaches have been described, such as the use of nested PCR which detects genus-specific genomic sequences or single-copy genes, the use of "panfungal" PCR which detects multiple-copy genes identifiable in many fungal species and is followed by hybridization with species-specific probes, and real-time PCR technologies such as LightCycler ${ }^{\mathrm{TM}}$ or TaqMan ${ }^{\mathrm{TM}}$ which even may allow the quantification of fungal burden.

A total of 11 studies of PCR using blood samples of pediatric cancer patients were analyzed in a recent systematic review (Lehrnbecher et al., 2016). Three of the studies enrolling a total of pediatric 147 patients were performed in a screening setting, whereas 8 studies enrolling a total of pediatric 539 patients evaluated PCR samples in children with high suspicion of IA, such as children with prolonged febrile neutropenia or children with lung infiltrates. In the screening setting, specificity, sensitivity, PPV, and NPV ranged from 43 to $85 \%, 11$ to $80 \%, 22$ to $50 \%$ and 60 to $96 \%$, respectively. In the diagnostic setting, specificity, sensitivity, PPV and NPV ranged from 36 to $83 \%, 0$ to $100 \%, 0$ to $71 \%$ and 88 to $100 \%$, respectively. Due to these results, a recent international expert group gave a strong recommendation with a moderate quality of evidence for not using fungal PCR testing in blood (Lehrnbecher et al., $2017 b$ ). Whether the combined use of GM and PCR improves the test results as it was shown in a study in adult high-risk hematological patients needs to be evaluated in the pediatric population (Morrissey et al., 2013).

As compared to blood, the performance of PCR in BAL samples seems to be superior. For example, it has been demonstrated in 226 adult patients that BAL PCR testing had a sensitivity of $69 \%$ and a specificity of $87 \%$ in patients who did not receive antifungals prior to BAL (Reinwald et al., 2012). Unfortunately, the performance of the test significantly decreased 
in patients who had received at least two antifungals, which may be the situation in a considerable proportion of pediatric patients prior to receiving a BAL.

It is important to mention that PCR evaluation of biopsy samples may have an important impact in the correct diagnosis and in the treatment of pulmonary infections with a fungal pathogen, as it was demonstrated in adult patients (Lass-Flörl et al., 2007). In addition, PCR based methods have also been investigated and are of major usefulness for the identification of mutations conferring resistance to specific antifungals (e.g., azole resistance in A. fumigatus) (White et al., 2015a).

\section{IMAGING STUDIES}

As autopsy data demonstrate that the lung is affected in almost $90 \%$ of the patients with IA (Groll et al., 1996; Lehrnbecher et al., 2010), imaging of the lung is an important tool in the early diagnosis of the infection. In adults, chest computerized tomography (CT) scans reveal pneumonia due to a fungal pathogen earlier as conventional chest radiographs (Heussel et al., 1999), which is important in order to institute early antifungal therapy which impacts upon outcome. In adults, CT findings such as the "halo sign," the "air-crescent sign", or "cavitation" have been included in the current EORTC/MSG definitions of IFD (Caillot et al., 1997; De Pauw et al., 2008). Nevertheless, these findings are not specific for pulmonary aspergillosis and may also be seen in other pulmonary infections or due to the progression and relapse of the underlying malignancy. In addition, the appearance of these signs depends on the time of imaging (Caillot et al., 1997).

Data on CT findings in children with IA are conflicting. One retrospective study in 139 children with IA demonstrated that the halo sign was seen in only $6.4 \%$ of the patients, the aircrescent sign in $1.6 \%$ of children and a cavitation in $14.4 \%$ of patients, respectively (Burgos et al., 2008). In contrast, nodules were found in $21 \%$ of the pediatric patients, corroborating the results of another report (Taccone et al., 1993). In contrast, a more recent pediatric study found that the halo sign in the chest CT was significantly associated with pulmonary IA (78.4 vs. $40.7 \%, P<0.001$ ) (Han et al., 2015). It is unclear whether the different results among the studies simply reflect different time points of CT imaging or are due to other, yet unknown reasons. Nevertheless, the appearance of any new infiltrate in the chest CT in children with prolonged febrile neutropenia not responding to broad-spectrum antibiotics should be considered as possible pulmonary fungal disease and trigger diagnostic procedures. Notably, in adults, high resolution CT angiography may improve radiological diagnosis by detecting vessel occlusions caused by invasive aspergillosis, but to date, no data are available in the pediatric population (Stanzani et al., 2012, 2015).

Regarding the radiologic evaluation of other sites in children with prolonged febrile neutropenia and a high suspicion of IFD, a recent pediatric guideline included a weak recommendation for routine abdominal imaging, as in four studies, imaging findings in a significant proportion of patients without localizing signs or symptoms were consistent with IFD (Bartley et al., 1982;
Archibald et al., 2001; Ahmad Sarji et al., 2006; Cohn et al., 2016; Lehrnbecher et al., 2017b). In contrast, the guideline panel gave a weak recommendation against routine sinus imaging in persistently febrile neutropenic patients in the absence of localizing signs or symptoms, which is based on the observation that sinus imaging is frequently abnormal in these patients and that the findings do not distinguish between children with and without invasive fungal infection of the sinus (Kavanagh et al., 1991; Archibald et al., 2001; Park et al., 2005; Ahmad Sarji et al., 2006; Cohn et al., 2016; Lehrnbecher et al., 2017b).

Whereas the lung is the primary portal of entry of Aspergillus, an autopsy study demonstrated that the infection is confined to the lung in only $41 \%$, but disseminated in $53 \%$ of the patients, and that the CNS is one of the most common sites of disseminated involvement (Groll et al., 1996). The clinical presentation of cerebral aspergillosis, which is associated with an extremely high mortality, depends on the site and extent of involvement and can be classified in a number of defined syndromes, such as fungal abscesses, granulomas, or cavitated lesions (Armenian et al., 2009; Castagnola et al., 2010). For these patients, magnetic resonance imaging (MRI) of the CNS is the most important diagnostic tool in order to identify and monitor cerebral fungal infections. There is an ongoing debate whether MRI of the CNS should routinely be performed in asymptomatic patients with pulmonary aspergillosis.

\section{INVASIVE DIAGNOSTIC PROCEDURES}

Invasive diagnostic procedures such as bronchoscopy combined with BAL or lung biopsy should be considered in the work-up of children with pulmonary infiltrates. Whereas, the isolation of Aspergillus spp. from the sputum of neutropenic patients with clinical symptoms consistent with IFD is only an indicator of fungal pneumonia, the histopathological identification and/or the culture of Aspergillus spp. from lung tissue remains the diagnostic "gold standard" for proof of pulmonary aspergillosis (Ruhnke et al., 2012).

A recent meta-analysis on the diagnostic yield and the complication rate of BAL and lung biopsies performed in both pediatric and adult patients with cancer or HSCT recipients included 72 studies on BAL and 31 studies on lung biopsies (Chellapandian et al., 2015). When comparing the two diagnostic tools, the proportion of procedures leading to any diagnosis including non-infectious diagnoses was similar. However, BAL led significantly more often to an infectious diagnosis than did lung biopsy ( 49 vs. $34 \% ; P<0.001$ ). A significant difference was seen for the identification of a bacterial infection, but not for the detection of viral and fungal infection, respectively. In contrast, lung biopsies resulted significantly more often in a non-infectious diagnosis than BAL (43 vs. $7 \% ; P<0.001$ ), but, at the same time, was associated with a significantly higher complication rate (15 vs. $8 \% ; P=0.006$ ). Whereas the diagnostic yield of BAL and lung biopsy seems to be similar between the pediatric and adult population, children had a higher complication rate during biopsy procedures than adults $(P=0.003)$. Studies 
which included the assessment of GM in BAL reported on a significantly higher detection rate of proven/probable IA than studies in which GM was not used (31\%; 95\% CI, $23-43 \%$ vs. $18 \%$; 95\% CI, 15-22\%; $P=0.005$ ), whereas the impact of PCR as a diagnostic tool in BAL samples is less clear. Notably, as mentioned above, the diagnostic performance of the PCR in BAL samples decreased in adult patients who had received at least two antifungal agents (Reinwald et al., 2012). In contrast, the diagnostic yield of invasive diagnostic procedures increased when different diagnostic tools (e.g., histopathology, antigen- and molecular testing) were combined (Lass-Flörl et al., 2007). On the other hand, according to a prospective study in 55 adult patients, a positive mycologic result in respiratory samples depended on the underlying malignancy and the leukocyte count, with significantly higher yields in non-acute leukemia and in patients with leukocyte counts of more than 100 per $\mu$ l (Bergeron et al., 2012). A meta-analysis showed that results of lung biopsies led significantly more often to a change in the clinical management ( $48 \%$ vs. $31 \%$; $P=0.002$ ), but it remains unclear whether this was associated with an improved overall outcome in this highly selected patient population.

\section{SUMMARY AND FUTURE PERSPECTIVES}

The early and reliable diagnosis of IA is difficult in immunocompromised patients, in particular in the pediatric population. In high risk patients with prolonged febrile neutropenia or signs and symptoms for IA, available diagnostic tools include imaging procedures and non-culture based techniques such as antigen-based and molecular biomarkers. The performance and clinical utility of these tests may be

\section{REFERENCES}

Ahmad Sarji, S., Wan Abdullah, W., and Wastie, M. (2006). Imaging features of fungal infection in immuno-suppressed patients in a local ward outbreak. Biomed. Imaging Interv. J. 2:e21. doi: 10.2349/biij.2.2.e21

Archibald, S., Park, J., Geyer, J. R., and Hawkins, D. S. (2001). Computed tomography in the evaluation of febrile neutropenic pediatric oncology patients. Pediatr. Infect. Dis. J. 20, 5-10. doi: 10.1097/00006454-200101000-00002

Armenian, S. H., Nash, K. A., Kapoor, N., Franklin, J. L., Gaynon, P. S., Ross, L. A., et al. (2009). Prospective monitoring for invasive aspergillosis using galactomannan and polymerase chain reaction in high risk pediatric patients. J. Pediatr. Hematol. Oncol. 31, 920-926. doi: 10.1097/MPH.0b013e3181b83e77

Bacher, P., Steinbach, A., Kniemeyer, O., Hamprecht, A., Assenmacher, M., Vehreschild, M. J., et al. (2015). Fungus-specific CD4(+) T cells for rapid identification of invasive pulmonary mold infection. Am. J. Respir. Crit. Care Med. 191, 348-352. doi: 10.1164/rccm.201407-1235LE

Badiee, P., Alborzi, A., Karimi, M., Pourabbas, B., Haddadi, P., Mardaneh, J., et al. (2012). Diagnostic potential of nested PCR, galactomannan EIA, and beta-Dglucan for invasive aspergillosis in pediatric patients. J. Infect. Dev. Ctries. 6, 352-357. doi: 10.3855/jidc. 2110

Bartley, D. L., Hughes, W. T., Parvey, L. S., and Parham, D. (1982). Computed tomography of hepatic and splenic fungal abscesses in leukemic children. Pediatr. Infect. Dis. 1, 317-321. doi: 10.1097/00006454-198209000-00007

Bergeron, A., Porcher, R., Sulahian, A., de Bazelaire, C., Chagnon, K., Raffoux, E., et al. (2012). The strategy for the diagnosis of invasive pulmonary aspergillosis should depend on both the underlying condition and the leukocyte different between children and adults, and they may also be different for invasive diagnostic procedures such as BAL or biopsy. However, it is important to note that considerably less data are available for children as compared to adults. Although the combination of diagnostic methods seems to improve sensitivity and specificity, new diagnostic tools are needed that will improve the early and reliable diagnosis of IA. In this respect, the lateral-flow device assay which is based on the JF5 antibody and detects an extracellular glycoprotein antigen secreted during active growth of Aspergillus spp, is a promising platform that is to be tested in the pediatric population, as it may allow for a rapid and simple bed-side testing of IA (Heldt and Hoenigl, 2017; Hoenigl et al., 2018). In addition, specific host response biomarkers may be an interesting approach to diagnose IA, as it has been demonstrated for the monitoring of Aspergillus-specific CD4 ${ }^{+}$T-cells (Bacher et al., 2015). Another interesting approach is the use of A. fumigatus-specific antibodies labeled with a radionuclide, which can be visualized by Positron Emission Tomography (PET)/MRI. Although not tested yet in humans, this approach could clearly distinguish invasive pulmonary aspergillosis from bacterial lung infection and other non-specific lung inflammation in the mouse model (Rolle et al., 2016). However, in order to perform meaningful studies to evaluate these diagnostic tools in the pediatric population, a comprehensive and necessarily international effort is necessary.

\section{AUTHOR CONTRIBUTIONS}

$\mathrm{TL}, \mathrm{AH}, \mathrm{AG}$, and $\mathrm{KB}$ designed the manuscript, wrote parts of the manuscript, reviewed, and approved the last version of the manuscript. count of patients with hematologic malignancies. Blood 119, 1831-1837. doi: 10.1182/blood-2011-04-351601

Burgos, A., Zaoutis, T. E., Dvorak, C. C., Hoffman, J. A., Knapp, K. M., Nania, J. J., et al. (2008). Pediatric invasive aspergillosis: a multicenter retrospective analysis of 139 contemporary cases. Pediatrics 121, e1286-e1294. doi: $10.1542 /$ peds.2007-2117

Caillot, D., Casasnovas, O., Bernard, A., Couaillier, J. F., Durand, C., Cuisenier, B., et al. (1997). Improved management of invasive pulmonary aspergillosis in neutropenic patients using early thoracic computed tomographic scan and surgery. J. Clin. Oncol. 15, 139-147. doi: 10.1200/JCO.1997. 15.1.139

Castagnola, E., Furfaro, E., Caviglia, I., Licciardello, M., Faraci, M., Fioredda, F., et al. (2010). Performance of the galactomannan antigen detection test in the diagnosis of invasive aspergillosis in children with cancer or undergoing haemopoietic stem cell transplantation. Clin. Microbiol. Infect. 16, 1197-1203. doi: 10.1111/j.1469-0691.2009.03065.x

Chai, L. Y., Kullberg, B. J., Johnson, E. M., Teerenstra, S., Khin, L. W., Vonk, A. G., et al. (2012). Early serum galactomannan trend as a predictor of outcome of invasive aspergillosis. J. Clin. Microbiol. 50, 2330-2336. doi: 10.1128/JCM.06513-11

Chellapandian, D., Lehrnbecher, T., Phillips, B., Fisher, B. T., Zaoutis, T. E., Steinbach, W. J., et al. (2015). Bronchoalveolar lavage and lung biopsy in patients with cancer and hematopoietic stem-cell transplantation recipients: a systematic review and meta-analysis. J. Clin. Oncol. 33, 501-509. doi: 10.1200/JCO.2014.58.0480

Cohn, S. M., Pokala, H. R., Siegel, J. D., McClay, J. E., Leonard, D., Kwon, J., et al. (2016). Application of a standardized screening protocol for diagnosis of 
invasive mold infections in children with hematologic malignancies. Support. Care Cancer 24, 5025-5033. doi: 10.1007/s00520-016-3367-z

de Mol, M., de Jongste, J. C., van Westreenen, M., Merkus, P. J., de Vries, A. H., Hop, W. C., et al. (2013). Diagnosis of invasive pulmonary aspergillosis in children with bronchoalveolar lavage galactomannan. Pediatr. Pulmonol. 48, 789-796. doi: 10.1002/ppul.22670

De Pauw, B., Walsh, T. J., Donnelly, J. P., Stevens, D. A., Edwards, J. E., Calandra, T., et al. (2008). Revised definitions of invasive fungal disease from the European Organization for Research and Treatment of Cancer/Invasive Fungal Infections Cooperative Group and the National Institute of Allergy and Infectious Diseases Mycoses Study Group (EORTC/MSG) Consensus Group. Clin. Infect. Dis. 46, 1813-1821. doi: 10.1086/ 588660

Desai, R., Ross, L. A., and Hoffman, J. A. (2009). The role of bronchoalveolar lavage galactomannan in the diagnosis of pediatric invasive aspergillosis. Pediatr. Infect. Dis. J. 28, 283-286. doi: 10.1097/INF.0b013e31818f0934

Fisher, B. T., Robinson, P. D., Lehrnbecher, T., Steinbach, W. J., Zaoutis, T. E., Phillips, B., et al. (2017). Risk factors for invasive fungal disease in pediatric cancer and hematopoietic stem cell transplantation: a systematic review. J. Pediatr. Infect. Dis. Soc. doi: 10.1093/jpids/pix030. [Epub ahead of print].

Goudjil, S., Kongolo, G., Dusol, L., Imestouren, F., Cornu, M., Leke, A., et al. (2013). (1-3)-beta-D-glucan levels in candidiasis infections in the critically ill neonate. J. Matern. Fetal Neonatal Med. 26, 44-48. doi: $10.3109 / 14767058.2012 .722716$

Groll, A. H., Castagnola, E., Cesaro, S., Dalle, J. H., Engelhard, D., Hope, W., et al. (2014). Fourth European Conference on Infections in Leukaemia (ECIL-4): guidelines for diagnosis, prevention, and treatment of invasive fungal diseases in paediatric patients with cancer or allogeneic haemopoietic stem-cell transplantation. Lancet Oncol. 15, e327-e340. doi: 10.1016/S1470-2045(14)70017-8

Groll, A. H., Shah, P. M., Mentzel, C., Schneider, M., Just-Nuebling, G., and Huebner, K. (1996). Trends in the postmortem epidemiology of invasive fungal infections at a university hospital. J. Infect. 33, 23-32. doi: 10.1016/S0163-4453(96)92700-0

Han, S. B., Kim, S. K., Bae, E. Y., Lee, J. W., Yoon, J. S., Chung, N. G., et al. (2015). Clinical features and prognosis of invasive pulmonary aspergillosis in korean children with hematologic/oncologic diseases. J. Korean Med. Sci. 30, 1121-1128. doi: 10.3346/jkms.2015.30.8.1121

Hassler, A., Porto, L., and Lehrnbecher, T. (2015). Cerebral fungal infection in pediatric cancer patients. Curr. Fungal Infect. Rep. 9, 6-14. doi: 10.1007/s12281-014-0213-y

Hayden, R. T., Isotalo, P. A., Parrett, T., Wolk, D. M., Qian, X., Roberts, G. D., et al. (2003). In situ hybridization for the differentiation of Aspergillus, Fusarium, and Pseudallescheria species in tissue section. Diagn. Mol. Pathol. 12, 21-26. doi: 10.1097/00019606-200303000-00003

Heldt, S., and Hoenigl, M. (2017). Lateral flow assays for the diagnosis of invasive aspergillosis: current status. Curr. Fungal Infect. Rep. 11, 45-51. doi: $10.1007 / \mathrm{s} 12281-017-0275-8$

Heussel, C. P., Kauczor, H. U., Heussel, G. E., Fischer, B., Begrich, M., Mildenberger, P., et al. (1999). Pneumonia in febrile neutropenic patients and in bone marrow and blood stem-cell transplant recipients: use of high-resolution computed tomography. J. Clin. Oncol. 17, 796-805. doi: 10.1200/JCO.1999.17.3.796

Hoenigl, M., Eigl, S., Heldt, S., Duettmann, W., Thornton, C., and Prattes, J. (2018). Clinical evaluation of the newly formatted lateral-flow device for invasive pulmonary aspergillosis. Mycoses 61, 40-43. doi: 10.1111/myc.12704

Hol, J. A., Wolfs, T. F., Bierings, M. B., Lindemans, C. A., Versluys, A. B., Wildt de A., et al. (2014). Predictors of invasive fungal infection in pediatric allogeneic hematopoietic SCT recipients. Bone Marrow Transplant. 49, 95-101. doi: 10.1038/bmt.2013.136

Kavanagh, K. T., Hughes, W. T., Parham, D. M., and Chanin, L. R. (1991). Fungal sinusitis in immunocompromised children with neoplasms. Ann. Otol. Rhinol. Laryngol. 100, 331-336. doi: 10.1177/0003489491100 00413

Koltze, A., Rath, P., Schöning, S., Steinmann, J., Wichelhaus, T. A., Bader, P., et al. (2015). Beta-D-Glucan screening for the detection of invasive fungal disease in children undergoing allogeneic hematopoietic stem cell transplantation. J. Clin. Microbiol. 53, 2605-2610. doi: 10.1128/JCM.00747-15
Kontoyiannis, D. P., Sumoza, D., Tarrand, J., Bodey, G. P., Storey, R., and Raad, I. I. (2000)., Significance of aspergillemia in patients with cancer: a 10-year study. Clin. Infect. Dis. 31, 188-189. doi: 10.1086/313918

Kovanda, L. L., Desai, A. V., and Hope, W. W. (2017). Prognostic value of galactomannan: current evidence for monitoring response to antifungal therapy in patients with invasive aspergillosis. J. Pharmacokinet. Pharmacodyn. 44, 143-151. doi: 10.1007/s10928-017-9509-1

Lamoth, F., Cruciani, M., Mengoli, C., Castagnola, E., Lortholary, O., Richardson, M., et al. L. (2012). Third European Conference on Infections in, beta-Glucan antigenemia assay for the diagnosis of invasive fungal infections in patients with hematological malignancies: a systematic review and meta-analysis of cohort studies from the Third European Conference on Infections in Leukemia (ECIL-3). Clin. Infect. Dis. 54, 633-643. doi: 10.1093/cid/cir897

Lass-Florl, C. (2017). Diagnosing fungal infections in haematology patientsanother case of less is more in the clinical setting? Clin. Microbiol. Infect. 23, 896-897. doi: 10.1016/j.cmi.2017.05.022

Lass-Flörl, C., Resch, G., Nachbaur, D., Mayr, A., Gastl, G., Auberger, J., et al. (2007). The value of computed tomography-guided percutaneous lung biopsy for diagnosis of invasive fungal infection in immunocompromised patients. Clin. Infect. Dis. 45, e101-e104. doi: 10.1086/521245

Lehrnbecher, T., Becker, K., and Groll, A. H. (2017a). Current algorithms in fungal diagnosis in the immunocompromised host. Methods Mol. Biol. 1508, 67-84. doi: 10.1007/978-1-4939-6515-1_3

Lehrnbecher, T., Frank, C., Engels, K., Kriener, S., Groll, A. H., and Schwabe, D. (2010). Trends in the postmortem epidemiology of invasive fungal infections at a university hospital. J. Infect. 61, 259-265. doi: 10.1016/j.jinf.2010.06.018

Lehrnbecher, T., Robinson, P. D., Fisher, B. T., Castagnola, E., Groll, A. H., Steinbach, W. J., et al. (2016). Galactomannan, beta-D-Glucan, and polymerase chain reaction-based assays for the diagnosis of invasive fungal disease in pediatric cancer and hematopoietic stem cell transplantation: a systematic review and meta-analysis. Clin. Infect. Dis. 63, 1340-1348. doi: 10.1093/cid/ciw592

Lehrnbecher, T., Robinson, P., Fisher, B., Alexander, S., Ammann, R. A., Beauchemin, M., et al. (2017b). Guideline for the management of fever and neutropenia in children with cancer and hematopoietic stem-cell transplantation recipients: 2017 update. J. Clin. Oncol. 35, 2082-2094. doi: 10.1200/JCO.2016.71.7017

Maertens, J., Maertens, V., Theunissen, K., Meersseman, W., Meersseman, P., Meers, S., et al. (2009). Bronchoalveolar lavage fluid galactomannan for the diagnosis of invasive pulmonary aspergillosis in patients with hematologic diseases. Clin. Infect. Dis. 49, 1688-1693. doi: 10.1086/647935

Maertens, J., Marchetti, O., Herbrecht, R., Cornely, O. A., Flückiger, U., Frêre, P., et al. (2010). European guidelines for antifungal management in leukemia and hematopoietic stem cell transplant recipients: summary of the ECIL 3-2009 Update. Bone Marrow Transplant. 46, 709-718. doi: 10.1038/bmt.2010.175

Maertens, J., Theunissen, K., Verbeken, E., Lagrou, K., Verhaegen, J., Boogaerts, M., et al. (2004). Prospective clinical evaluation of lower cutoffs for galactomannan detection in adult neutropenic cancer patients and haematological stem cell transplant recipients. Br. J. Haematol. 126, 852-860. doi: 10.1111/j.1365-2141.2004.05140.x

Maertens, J., Theunissen, K., Verhoef, G., Verschakelen, J., Lagrou, K., Verbeken, E., et al. (2005). Galactomannan and computed tomography-based preemptive antifungal therapy in neutropenic patients at high risk for invasive fungal infection: a prospective feasibility study. Clin. Infect. Dis. 41, 1242-1250. doi: $10.1086 / 496927$

Maertens, J., Verhaegen, J., Demuynck, H., Brock, P., Verhoef, G., Vandenberghe, P., et al. (1999). Autopsy-controlled prospective evaluation of serial screening for circulating galactomannan by a sandwich enzyme-linked immunosorbent assay for hematological patients at risk for invasive Aspergillosis. J. Clin. Microbiol. 37, 3223-3228.

Maertens, J., Verhaegen, J., Lagrou, K., Van Eldere, J., and Boogaerts, M. (2001). Screening for circulating galactomannan as a noninvasive diagnostic tool for invasive aspergillosis in prolonged neutropenic patients and stem cell transplantation recipients: a prospective validation. Blood 97, 1604-1610. doi: 10.1182/blood.V97.6.1604

Maschmeyer, G., Carratalà, J., Buchheidt, D., Hamprecht, A., Heussel, C. P., Kahl, C., et al. (2015). Diagnosis and antimicrobial therapy of lung infiltrates in febrile neutropenic patients (allogeneic SCT excluded): updated guidelines 
of the Infectious Diseases Working Party (AGIHO) of the German Society of Hematology and Medical Oncology (DGHO). Ann. Oncol. 26, 21-33. doi: 10.1093/annonc/mdu192

Morrissey, C. O., Chen, S. C., Sorrell, T. C., Milliken, S., Bardy, P. G., Bradstock, K. F., et al. G. (2013). Australasian leukaemia lymphoma, a. the, and G. New Zealand mycology interest, galactomannan and PCR versus culture and histology for directing use of antifungal treatment for invasive aspergillosis in high-risk haematology patients: a randomised controlled trial. Lancet Infect. Dis. 13, 519-528. doi: 10.1016/S1473-3099(13)70076-8

Naselli, A., Faraci, M., Lanino, E., Morreale, G., Cangemi, G., Bandettini, R., et al. (2015). Persistence of high-level (1,3)-beta-D-glucan after candidemia following autologous peripheral SCT in a pediatric patient. Bone Marrow Transplant. 50, 137-138. doi: 10.1038/bmt.2014.206

Nouér, S. A., Nucci, M., Kumar, N. S., Grazziutti, M., Barlogie, B., and Anaissie, E. (2011). Earlier response assessment in invasive aspergillosis based on the kinetics of serum Aspergillus galactomannan: proposal for a new definition. Clin. Infect. Dis. 53, 671-676. doi: 10.1093/cid/cir441

Oz, Y., and Kiraz, N. (2011). Diagnostic methods for fungal infections in pediatric patients: microbiological, serological and molecular methods. Expert Rev. Anti Infect. Ther. 9, 289-298. doi: 10.1586/eri.10.168

Pagano, L., Caira, M., Nosari, A., Van Lint, M. T., Candoni, A., Offidani, M., et al. (2007). Fungal infections in recipients of hematopoietic stem cell transplants: results of the SEIFEM B-2004 study-Sorveglianza Epidemiologica Infezioni Fungine Nelle Emopatie Maligne. Clin. Infect. Dis. 45, 1161-1170. doi: $10.1086 / 522189$

Park, A. H., Muntz, H. R., Smith, M. E., Afify, Z., Pysher, T., and Pavia, A. (2005). Pediatric invasive fungal rhinosinusitis in immunocompromised children with cancer. Otolaryngol. Head Neck Surg. 133, 411-416. doi: 10.1016/j.otohns.2005.04.014

Reinwald, M., Hummel, M., Kovalevskaya, E., Spiess, B., Heinz, W. J., Vehreschild, J. J., et al. (2012). Therapy with antifungals decreases the diagnostic performance of PCR for diagnosing invasive aspergillosis in bronchoalveolar lavage samples of patients with haematological malignancies. J. Antimicrob. Chemother. 67, 2260-2267. doi: 10.1093/jac/dks208

Reischies, F. M., Raggam, R. B., Prattes, J., Krause, R., Eigl, S., List, A., et al. (2016). Urine galactomannan-to-creatinine ratio for detection of invasive aspergillosis in patients with hematological malignancies. J. Clin. Microbiol. 54, 771-774. doi: 10.1128/JCM.02969-15

Roilides, E., Pavlidou, E., Papadopoulos, F., Panteliadis, C., Farmaki, E., Tamiolaki, M., et al. (2003). Cerebral aspergillosis in an infant with corticosteroid-resistant nephrotic syndrome. Pediatr. Nephrol. 18, 450-453. doi: 10.1007/s00467-003-1113-5

Rolle, A. M., Hasenberg, M., Thornton, C. R., Solouk-Saran, D., Männ, L., Weski, J., et al. (2016). ImmunoPET/MR imaging allows specific detection of Aspergillus fumigatus lung infection in vivo. Proc. Natl. Acad. Sci. U.S.A. 113, E1026E1033. doi: 10.1073/pnas.1518836113

Ruhnke, M., Bohme, A., Buchheidt, D., Cornely, O., Donhuijsen, K., Einsele, H., et al. (2012). Infectious diseases working party in, H. oncology of the german society for, and oncology, diagnosis of invasive fungal infections in hematology and oncology-guidelines from the infectious diseases working party in haematology and oncology of the german society for haematology and oncology (AGIHO). Ann. Oncol. 23, 823-833. doi: 10.1093/annonc/mdr407

Science, M., Robinson, P. D., MacDonald, T., Rassekh, S. R., Dupuis, L. L., and Sung, L. (2014). Guideline for primary antifungal prophylaxis for pediatric patients with cancer or hematopoietic stem cell transplant recipients. Pediatr. Blood Cancer 61, 393-400. doi: 10.1002/pbc.24847

Smith, P. B., Benjamin, D. K. Jr., Alexander, B. D., Johnson, M. D., Finkelman, M. A., and Steinbach, W. J. (2007). Quantification of 1,3-beta$\mathrm{D}$-glucan levels in children: preliminary data for diagnostic use of the betaglucan assay in a pediatric setting. Clin. Vaccine Immunol. 14, 924-925. doi: 10.1128/CVI.00025-07
Stanzani, M., Battista, G., Sassi, C., Lewis, R. E., Tolomelli, G., Clissa, C., et al. (2012). Computed tomographic pulmonary angiography for diagnosis of invasive mold diseases in patients with hematological malignancies. Clin. Infect. Dis. 54, 610-616. doi: 10.1093/cid/cir861

Stanzani, M., Sassi, C., Lewis, R. E., Tolomelli, G., Bazzocchi, A., Cavo, M., et al. (2015). High resolution computed tomography angiography improves the radiographic diagnosis of invasive mold disease in patients with hematological malignancies. Clin. Infect. Dis. 60, 1603-1610. doi: 10.1093/cid/civ154

Sulahian, A., Boutboul, F., Ribaud, P., Leblanc, T., Lacroix, C., and Derouin, F. (2001). Value of antigen detection using an enzyme immunoassay in the diagnosis and prediction of invasive aspergillosis in two adult and pediatric hematology units during a 4-year prospective study. Cancer 91, 311-318. doi: 10.1002/1097-0142(20010115)91:2<311::AID-CNCR1003>3.0.CO;2-3

Sung, L., Lange, B. J., Gerbing, R. B., Alonzo, T. A., and Feusner, J. (2007). Microbiologically documented infections and infection-related mortality in children with acute myeloid leukemia. Blood 110, 3532-3539. doi: 10.1182/blood-2007-05-091942

Taccone, A., Occhi, M., Garaventa, A., Manfredini, L., and Viscoli, C. (1993). CT of invasive pulmonary aspergillosis in children with cancer. Pediatr. Radiol. 23, 177-180. doi: 10.1007/BF02013825

Upton, A., Kirby, K. A., Carpenter, P., Boeckh, M., and Marr, K. A. (2007). Invasive aspergillosis following hematopoietic cell transplantation: outcomes and prognostic factors associated with mortality. Clin. Infect. Dis. 44, 531-540. doi: $10.1086 / 510592$

Viscoli, C., Machetti, M., Cappellano, P., Bucci, B., Bruzzi, P., Van Lint, M. T., et al. (2004). False-positive galactomannan platelia Aspergillus test results for patients receiving piperacillin-tazobactam. Clin. Infect. Dis. 38, 913-916. doi: $10.1086 / 382224$

Viscoli, C., Machetti, M., Gazzola, P., De Maria, A., Paola, D., Van Lint, M. T., et al. (2002). Aspergillus galactomannan antigen in the cerebrospinal fluid of bone marrow transplant recipients with probable cerebral aspergillosis. J. Clin. Microbiol. 40, 1496-1499. doi: 10.1128/JCM.40.4.14961499.2002

White, P. L., Bretagne, S., Klingspor, L., Melchers, W. J., McCulloch, E., Schulz, B., et al. (2010). Aspergillus PCR: one step closer to standardization. J. Clin. Microbiol. 48, 1231-1240. doi: 10.1128/JCM.01767-09

White, P. L., Posso, R. B., and Barnes, R. A. (2015a). Analytical and clinical evaluation of the pathonostics aspergenius assay for detection of invasive aspergillosis and resistance to azole antifungal drugs during testing of serum samples. J. Clin. Microbiol. 53, 2115-2121. doi: 10.1128/JCM.00667-15

White, P. L., Wingard, J. R., Bretagne, S., Loffler, J., Patterson, T. F., Slavin, M. A., et al. (2015b). Aspergillus PCR: a systematic review of evidence for clinical use in comparison with antigen testing. Clin. Infect. Dis. 61, 1293-1303. doi: $10.1093 / \mathrm{cid} / \operatorname{civ} 507$

Zhao, L., Tang, J. Y., Wang, Y., Zhou, Y. F., Chen, J., Li, B. R., et al. (2009). Value of plasma $\beta$-Glucan in early diagnosis of invasive fungal infection in children. Chin. J. Contemp. Pediatr. 11, 905-908.

Conflict of Interest Statement: The authors declare that the research was conducted in the absence of any commercial or financial relationships that could be construed as a potential conflict of interest.

The reviewer VS and handling Editor declared their shared affiliation.

Copyright (C) 2018 Lehrnbecher, Hassler, Groll and Bochennek. This is an open-access article distributed under the terms of the Creative Commons Attribution License (CC $B Y)$. The use, distribution or reproduction in other forums is permitted, provided the original author(s) and the copyright owner are credited and that the original publication in this journal is cited, in accordance with accepted academic practice. No use, distribution or reproduction is permitted which does not comply with these terms. 